Published in final edited form as:

Helicobacter. 2008 October ; 13(5): 352-360. doi:10.1111/j.1523-5378.2008.00624.x.

\title{
Helicobacter Pylori and Gastroesophageal Reflux Disease:
}

\author{
A Community-Based Study \\ Douglas A. Corley, MD, PhD ${ }^{1,2}$, Ai Kubo, $\mathrm{PhD}^{1,3}$, T.R. Levin, MD ${ }^{1}$, Gladys Block, PhD ${ }^{4}$, \\ Laurel Habel, PhD $^{1}$, Gregory Rumore, MD ${ }^{1}$, Charles Quesenberry, PhD $^{1}$, Patricia Buffler, \\ $\mathrm{PhD}^{4}$, and Julie Parsonnet, $\mathbf{M D}^{5}$ \\ ${ }^{1}$ Kaiser Permanente; Division of Research \\ ${ }^{2}$ University of California, San Francisco; Department of Medicine and Comprehensive Cancer \\ Center \\ ${ }^{3}$ Columbia University, Mailman School of Public Health \\ ${ }^{4}$ University of California, Berkeley; School of Public Health \\ ${ }^{5}$ Stanford University, Department of Medicine and Department of Health Research and Policy
}

\begin{abstract}
Background-Gastric colonization with Helicobacter pylori is a proposed protective factor against gastroesophageal reflux disease (GERD), but little population-based data exist and other data conflict.

Methods-We conducted a community-based case-control study that compared GERD-free subjects with two groups: 1) subjects with a physician-assigned GERD diagnosis and 2) general population subjects with self-described weekly GERD symptoms. Subjects completed interviews, GERD questionnaires, and antibody testing for Helicobacter pylori and its cagA protein.
\end{abstract}

Results-Serologic data was available for 301 physician-assigned GERD patients, 81 general population patients with GERD symptoms, and 175 subjects from the general population without GERD. Physician-assigned GERD patients were less likely to have Helicobacter pylori antibodies than GERD-free population controls (odds ratio $[\mathrm{OR}]=0.27,95 \%$ confidence interval $[\mathrm{CI}]$ 0.15-0.47); there was also an inverse association between Helicobacter pylori and GERD symptom severity $(\mathrm{OR}=0.18,95 \% \mathrm{CI} 0.08-0.41$; severe or very severe symptoms) and GERD frequency $(\mathrm{OR}=0.18,95 \% \mathrm{CI} 0.09-0.38$; for symptoms at least weekly). The association was stronger among persons with erosive GERD and was similar between Helicobacter pylori positive subjects with and without cagA. There was no association among persons who were cagA positive, but Helicobacter pylori negative. Similar findings were found in analyses of population members with self-described GERD symptoms.

Conclusions-Helicobacter pylori antibody status was inversely associated with a GERD diagnosis and GERD symptoms in a community-based population.

\section{Keywords}

Helicobacter pylori; gastroesophageal reflux; GERD

Funding Sources: United States National Institutes of Health RO1 DK63616 and K08 DK02697. The study sponsor reviewed the protocol, but did not participate in the collection, analysis, or interpretation of the data.

Conflict of Interest: Dr. Corley and the other authors have no conflicts to declare. 


\section{BACKGROUND}

Gastroesophageal reflux disease (GERD) is a common, morbid, and costly medical condition in many countries.(1-4) Despite the prevalence of the disease, little is known regarding why only some persons develop GERD or GERD-related complications; infectious agents such as Helicobacter pylori are one proposed individual-level factor.

Helicobacter pylori (H. pylori) are bacteria that frequently colonize the lining of the stomach. An inverse association between H. pylori and GERD is often suggested; however, high-quality data, population-based data bolstering this purported link is minimal and discordant:(5-13) two recent systematic reviews had heterogeneous results and neither contained any studies (outside of Asia) with true population control groups and a recent population-based study in Norway showed no association between H.pylori and GERD symptoms.(13-15) One possible reason for the disparate results is the choice of control groups. Almost all studies extant examined series of endoscopy patients with GERD or GERD complications, and compared them with patients receiving an endoscopy for another indication. $(6,12,16-22)$ Because most control subjects had a reason for the endoscopy, these results may be biased. Patients with nonulcer dyspepsia or peptic ulcer disease, for example, may be more likely to be colonized with $H$. pylori than the general population.(23-25) Thus, comparing subjects with GERD vs. endoscopy controls without GERD may suggest that the prevalence of Helicobacter pylori infection is lower among GERD patients when, in fact, it is the non-GERD group undergoing endoscopy that has a higher than average prevalence. In addition, chronic intestinal symptoms (such as GERD) may lead to the treatment of $H$. pylori, leading to negative biopsies for $H$. pylori or for a negative serology for $H$. pylori at the time of the endoscopy, even if the person had been previously infected, leading to falsely low levels of $H$. pylori prevalence among the GERD patients.(25) No studies we identified controlled for prior treatment of $H$. pylori. Several adverse effects have been associated with colonization by $H$. pylori, including gastritis, peptic ulcer disease, and gastric cancer. The absence of potentially beneficial effects from $\mathrm{H}$. pylori has led to recommendations for its eradication, even among persons without demonstrated disease.(26,27) The use of population-based controls and data regarding prior treatment status would permit a better evaluation of any possible association between $H$. pylori and GERD, and would inform discussions regarding routine $H$. pylori testing and eradication.

We evaluated the associations between Helicobacter pylori antibody status and GERD symptoms in a community-based population to evaluate links between Helicobacter pylori and GERD.

\section{DESIGN AND METHODS}

\section{Study Population}

We conducted a case-control study within the Kaiser Permanente, Northern California (KPNC) integrated health services delivery organization. KPNC contains approximately 3.3 million persons and its membership demographics closely approximate the underlying census population of Northern California.(28) Eligible subjects were all adult (ages 18-79 years) members with at least 2 years membership prior to their index date, met the group definitions outlined below, and understood spoken and written English. We used data from two control groups originally recruited for a case-control study of Barrett's esophagus: a group of patients with a physician-assigned GERD diagnosis who had undergone esophagogastroduodenoscopy and a randomly selected population control group, both of whom were serially recruited between October, 2002 and September, 2005. Details have been previously published.(29) The physician-assigned GERD group and the population control groups were originally frequency matched by gender, age at the index date of the 
Barrett's esophagus cases, and geographic region (each subject's home facility) to subjects with a new diagnosis of Barrett's esophagus; subjects were serially enrolled using incidence density sampling, which matches eligible controls to the cases at the time of case diagnosis. For the first analysis in the current study, we contrasted the prevalence of $H$. pylori between members of the physician-assigned GERD group and "GERD-free" members of the general population sample (heartburn or acid reflux $<1 /$ month). Given patients undergoing endoscopy may differ from the average patient with GERD, we performed a second analysis confined to the population control group; that analysis compared population controls with GERD symptoms vs. GERD-free members of the general population sample.

\section{GERD Group (physician-assigned)}

GERD group members were randomly selected from among persons in the membership who had all of the following characteristics prior to their index date: a GERD-related diagnosis code (ICD-9 codes 530.11 [reflux esophagitis] or 530.81 [gastroesophageal reflux]); a prescription for at least 90 days supply of a histamine-2 receptor antagonist or a proton pump inhibitor (medications used for treating GERD symptoms) in the year prior to their index date (from electronic pharmacy records); and an esophagogastroduodenoscopy near the index date that did not demonstrate esophageal columnar metaplasia (e.g. Barrett's esophagus) of any length, by endoscopic appearance or pathology. Separate from the physician assignment, 94\% of these physician-assigned GERD subjects described at interview at least occasional GERD symptoms, and $81 \%$ described at least weekly symptoms.

\section{Population Controls}

Controls from the base population were randomly selected from among members of the entire Northern California Kaiser Permanente membership roster who lacked a prior diagnosis of Barrett's esophagus.(30)

\section{Exposure Measurements}

The H. pylori assays used in-house ELISA assays that have been extensively used in the Kaiser Permanente population and validated in different ethnic groups.(31-33) The assay's sensitivity and specificity for a current, active infection (compared to histopathology) have been $94 \%$ and $91 \%$, respectively. All subjects were also tested for antibodies to the H. pylori CagA protein (OraVax, Inc, Cambridge, MA).(34) Laboratory personnel were blinded to the case status and the samples were run in mixed batches of GERD patients and population controls.

\section{Outcome and Confounder Measurements}

All study subjects completed: an in-person interview (most commonly at the subject's home) of GERD symptoms, medication use, and medical history; a food frequency questionnaire; phlebotomy; and anthropometric measurements. Participants reported symptoms, diet, and other exposures in the year prior to the index date.

GERD symptom frequency and severity were evaluated with a validated questionnaire.(35) GERD was defined as heartburn (a burning pain or discomfort behind the breastbone) or acid regurgitation (a bitter or sour-tasting fluid coming up into the throat or mouth). Severity was recorded as mild (could be ignored), moderate (could not be ignored, but didn't affect lifestyle), severe (could not be ignored and did affect lifestyle), or very severe (markedly affected lifestyle). Frequency was defined as never, less than once a month, once a month, once a week, several times a week, or daily. 


\section{Confounding and Effect Modification}

We evaluated the following as potential confounders: BMI, ethnicity (Caucasian vs. nonCaucasian), smoking, recent alcohol use, aspirin or nonsteroidal anti-inflammatory drug (NSAID) use (including over-the-counter use), a comorbidity index (the DxCg score), calorie intake, waist circumference, socioeconomic data (education grade level and household income), and multivitamin use.

\section{Statistical Analysis}

We performed two main analyses: first, we compared the physician-assigned GERD group with GERD-free population controls (the primary analysis); second, we contrasted population controls with GERD symptoms at least weekly with the GERD-free population controls (the secondary analysis). We utilized standard analytic techniques for case-control studies including unconditional logistic regression.(30,36-38) Confounders were incorporated if their inclusion altered the odds ratio for the main effects by $>10 \%$ (e.g. education level) or if they were a frequency matched variable (gender, age, and medical facility). We evaluated for effect modification (e.g. differences in the associations by gender, smoking or BMI) by fitting cross product terms in the logistic regression model and by contrasting stratum specific odds ratios.(38) Associations were considered to happen by greater than chance if the $95 \%$ confidence interval excluded 1.0.

The study and analyses were approved by the institutional review board and all subjects provided written informed consent. Analyses used the STATA statistical package (version 8, STATA Corporation, College Station, TX).

\section{RESULTS}

\section{Study Population}

We interviewed 316 GERD patients and 317 population control subjects for the original study; serologic data were available for 611 subjects (97\% of interviewed subjects): 312 GERD patients, and 299 population controls. After exclusion of subjects with equivocal $H$. pylori assays, there were 301 physician-assigned GERD patients and 295 controls. Of these population controls, 175 (59\%) reported heartburn or acid regurgitation less than once a month (the GERD-free control group for the analyses), 81 (27\%) had at least weekly GERD symptoms (these subjects formed the self-described GERD group from the population controls), 38 described a GERD symptom only once a month (these subjects were not included in either group), and one had an incomplete response. The general subject characteristics are provided in Table 1. Positive antibody titers for $H$. pylori were found in 29 members (9.6\%) of the physician-defined GERD group, 15 (18.5\%) of the population controls with at least weekly GERD symptoms, and 44 (25.1\%) of the population controls without GERD symptoms, respectively.

\section{PHYSICIAN-DEFINED GERD GROUP}

Helicobacter pylori antibody status-Members of the physician-defined GERD group were significantly less likely to have a positive $H$. pylori antibody status compared to the population controls without GERD symptoms (OR 0.27, 95\% CI 0.15-0.47) (Table 2).

Gastroesophageal Reflux Symptoms-There was an inverse association between a positive $H$. pylori antibody status and GERD symptom frequency and severity (Table 3). For the comparison of the physician-defined GERD group vs. population controls without GERD symptoms, subjects with a positive H. pylori antibody status were much less likely to 
have severe or very severe GERD symptoms ( $\mathrm{OR}=0.18,95 \% \mathrm{CI} 0.08-0.41)$ or frequent symptoms (GERD symptoms several times a week or daily OR $=0.18,95 \%$ CI $0.09-0.38$ ).

Erosive GERD-We evaluated whether the association between $H$. pylori and GERD differed between patients with vs. without evidence of erosive GERD. Among the 35 members of the physician-defined GERD group $(12 \%)$ with visible esophageal ulcers, erosions, or strictures at the time of their endoscopy, none had $H$. pylori antibodies; this precluded calculation of an exact odds ratio. In this circumstance, an approximate odds ratio can be estimated by assuming a small value (such as 0.5 or 1.0) for the null value;(36) assuming one erosive GERD patient had $\mathrm{H}$. pylori provided an unadjusted $\mathrm{OR}=0.08,95 \%$ CI 0.00-0.54 for the association between H. pylori and erosive GERD. There was also a somewhat weaker inverse association among GERD group members without erosive GERD (OR=0.38 95\% CI 0.23-0.64).

CagA+ status-The inverse association between $H$. pylori and the physician-defined GERD group was similar between $H$. pylori antibody positive subjects with cagA+ strains $(\mathrm{OR}=0.26,95 \% \mathrm{CI} 0.11-0.62)$ and those without cagA $(\mathrm{OR}=0.25,95 \%$ CI $0.13-0.49)$ (Table 2). The presence of cagA antibodies was not associated with GERD frequency or severity (Table 3). There was no association among persons who were cagA positive, but Helicobacter pylori negative.

\section{GENERAL POPULATION GERD GROUP}

All physician-defined GERD group members received an esophagogastroduodenoscopy. Although this is similar to most $H$. pylori studies, patients undergoing endoscopy may differ from a more general population of GERD patients. We thus evaluated the association between $H$. pylori antibody and GERD symptoms among only the population controls (i.e. comparing the 175 GERD-free members of population group with the 81 members of the population group who experienced at least weekly GERD symptoms). Subjects with at least weekly GERD symptoms were much less likely than GERD-free controls to have a positive H. pylori antibody status (OR=0.46, 95\% CI 0.23-0.95) (Table 4). Inverse association patterns were also seen between $H$. pylori and GERD symptom severity (severe/very severe GERD symptoms OR=0.41, 95\% CI 0.14-1.18) and symptom frequency (GERD at least weekly $\mathrm{OR}=0.46,95 \% \mathrm{CI} 0.23-0.95$ ). Among persons who were $H$. pylori antibody positive, a cagA+ status did not influence the risk beyond that of being $H$. pylori positive.

\section{CONFOUNDING AND SUPPLEMENTAL ANALYSES}

There was no evidence of confounding by BMI, waist circumference, caloric intake, smoking status, alcohol use, aspirin use, nonsteroidal anti-inflammatory agents, ethnicity, household income, multivitamin use, or comorbidity status (DxCg score). The exclusion of educational status had a small influence on the primary analysis; thus, this was included in the main models in addition to the matched variables of age, gender, and facility. A fully adjusted model containing all the listed factors (plus age, gender, and facility) $(\mathrm{OR}=0.22$, $95 \%$ CI $0.12,0.42$ ) was very similar to a model that contained only the bivariate association between a physician-assigned GERD status and $H$. pylori antibody status $(\mathrm{OR}=0.32,95 \% \mathrm{CI}$ $0.19,0.55)$.

The inverse association between a positive $H$. pylori antibody and a physician-defined GERD status was slightly different between persons at different levels of BMI, though there was no statistical evidence of interaction ( $\mathrm{p}$-value on interaction term for BMI categories $=0.13$ ) and no clear trend with increasing $\mathrm{BMI}$ (for $\mathrm{BMI}<25, \mathrm{OR}=0.30,95 \% \mathrm{CI}$ 0.10-0.85; for BMI 25-29, OR=0.39, 95\% CI 0.15-1.04; for BMI $>=30, \mathrm{OR}=0.14,95 \% \mathrm{CI}$ $0.05-0.42)$. There were no differences between subjects who had ever smoked ( $\mathrm{OR}=0.24$, 
95\% CI 0.12-0.50) vs. nonsmokers ( $\mathrm{OR}=0.30,95 \%$ CI 0.12-0.74); similar findings were seen for current smokers.

Although GERD symptoms are not typically treated with effective H.pylori eradication regimens, we evaluated for reverse causation whereby GERD symptoms resulted in taking medications (prior to the index endoscopy) that might decrease the H. pylori antibody titer at the time of phlebotomy. Given antibody titers fall slowly, we only evaluated treatments prescribed greater than one year prior to the index dates.(39) Thirty-five subjects received effective two or three drug anti-H.pylori antibiotic regimens (32 physician-defined GERD patients [10\%] vs. 3 GERD-free controls [2\%]); the inverse association between a positive H. pylori antibody and GERD persisted after exclusion of these previously treated subjects $(\mathrm{OR}=0.25,95 \%$ CI $0.14-0.45)$.

Sixteen persons were $H$. pylori negative but cagA positive; the interpretation of this pattern is unclear and subjects with these findings are often excluded or analyzed separately. $(40,41)$ Based on an a priori (pre-analysis) strategy, we reported results for this group separately. Pooling together all $\mathrm{H}$. pylori and all cagA positive subjects as being "positive" for $H$. pylori decreased but did not eliminate a significant association between $H$. pylori and a physicianassigned GERD diagnosis ( $\mathrm{OR}=0.39,95 \%$ CI $0.24-0.50)$; for the general population GERD group, the $95 \%$ confidence interval included $1.0(\mathrm{OR}=0.55,95 \% \mathrm{CI} 0.28-1.09)$ for this analysis.

We evaluated whether the associations found were similar in different demographic groups. There were similar associations between $H$. pylori status and GERD symptoms among both men $(\mathrm{OR}=0.31,95 \% \mathrm{CI} 0.16-0.60)$ and women $(\mathrm{OR}=0.20$, 95\% CI 0.7-0.56). The association was possibly weaker among younger persons (e.g. ages $40-59$ years, $\mathrm{OR}=0.66$, 95\% CI 0.18-2.43 vs. ages 60-79 years $\mathrm{OR}=0.20,95 \%$ CI 0.11-0.39), although there was no statistical evidence of interaction by age as a continuous or categorical variable (p-value on interaction term for age as a continuous variable $=0.15$ ).

\section{DISCUSSION}

To our knowledge, this is the first study of the association between Helicobacter pylori antibody status and GERD using population-based controls in the United States. We found that the presence of antibodies against $H$. pylori had a strong inverse association with GERD symptom frequency and severity. These analyses suggest that, if the associations are causal, a portion of the risk for GERD may be associated with the absence of gastric colonization with the Helicobacter pylori bacterium.

This study extends the findings of previous analyses, which also suggested that persons without Helicobacter pylori colonization were more likely to have GERD symptoms, although prior studies have had conflicting results. The primary methodological difference in the current study is its use of a random sample of population-based controls (rather than endoscopy controls); this permits a less biased estimate of the association between $\mathrm{H}$. pylori and GERD. Prior meta-analyses of the associations between H. pylori and GERD have demonstrated marked heterogeneity, with some studies demonstrating no association. $(14,15)$ The studies outside Asia have consisted almost exclusively of endoscopy studies that lacked true population-based controls; in these studies, GERD patients were typically contrasted with patients referred for other gastrointestinal problems, possibly contributing to the conflicting results.(6,42-44) A recent large (604 patients) cross-sectional study among endoscopy patients actually suggested an increased risk of Barrett's oesophagus and GERD among patients with $\mathrm{H}$. pylori;(16) in contrast, a recent study by our group found an inverse association between $\mathrm{H}$. pylori (including cagA+ strains) and Barrett's esophagus.(45) 
Population-based studies in Norway and Sweden, which also assayed for a potential marker for gastric atrophy, did not find an association between H.pylori and GERD; the authors attributed this possibly to the low prevalence of gastric atrophy in their population.(13,41) The main finding of the current study, that the absence of $H$. pylori was associated with GERD symptoms, also differs from most randomized treatment trials of $H$. pylori, which indicate there is no increase in GERD symptoms after $H$. pylori eradication. These disparate results, however, are not incompatible.(10) $H$. pylori infection most commonly first occurs during early childhood, $(46,47)$ and the resultant long-standing bacterial colonization with $H$. pylori may induce gastric mucosal atrophy and decreased gastric acid secretion, with a related reduction in acid reflux into the esophagus. $(34,41,48,49)$ These changes may not be rapidly or fully reversible with the eradication of $H$. pylori in adults; acid production may take years after eradication to resume (or may never return to normal) and an increase in GERD symptoms may not have been detected in short-term follow-up studies, even though long-term H. pylori infection may still influence the risk of GERD compared with long-term uninfected subjects. These findings underscore the complex biologic role of H.pylori, given its known other positive associations with gastric cancer and peptic ulcer disease, $(26,50,51)$ and its inverse associations with esophageal adenocarcinoma (possibly mediated by GERD) in population-based studies. $(41,52,53)$

The interpretation of persons who are H. pylori negative but cagA positive is unclear. A reliability study found that a CagA antibody test was reproducible $93.4 \%$ of the time for persons with a positive $H$. pylori result and $86.3 \%$ of the time for persons with negative $H$. pylori antibodies.(54) Thus, some such results may be false positives, yet this antibody pattern may also be associated with gastric atrophy and gastric cancer, similar to persons who are $H$. pylori positive. $(41,55)$ A population-based study of esophageal adenocarcinoma, a disease strongly associated with gastroesophageal reflux disease, found no association between persons who were cagA+ but $H$. pylori, similar to the results found in the current study for GERD.(41) That study, however, also found an association between the cagA $+/ H$ Pylori negative antibody pattern and esophageal squamous cell carcinoma, even though no association was present in their adjusted analyses between $H$. pylori antibodies and esophageal squamous cell carcinoma. Thus, persons who are $H$. pylori negative but cagA positive may have had remote infections, produced false positive cagA results, have a different disease biology, or incorporate a combination of these factors.

There are several potential mechanisms through which the absence of Helicobacter pylori colonization may be associated with GERD. First and foremost, Helicobacter pylori infection has been postulated to cause gastric atrophy and directly suppress gastric acid production, particularly in patients with infections that are predominantly in the gastric body.(34,41,48,49,56,57) This effect is variable, however, and some gastric patterns of infection may actually result in increased acid production. $(56,57)$ Second, the absence of $H$. pylori may also be associated with increased appetite and a higher BMI, which is a separate risk factor for GERD, although adjustment for BMI did not influence the associations seen in the current study. Third, the results could also be explained if the gastric environment of persons who develop GERD is less hospitable to the establishment of $\mathrm{H}$. pylori, although data on this theory is minimal.

There are several strengths of this analysis. First, we found similar results in two different groups of GERD patients: one group subject to intensive investigation (the GERD group who underwent endoscopy) and a separate group of GERD patients from a population sample. This approach provided a group with a clinical diagnosis of GERD (a physicianassigned GERD diagnosis code plus the use of acid suppressing medications) and a group diagnosed from symptoms only (the population control group with self-described GERD symptoms). Both groups were community-based random samples of all persons who met the 
eligibility criteria; thus, the subjects were less susceptible to selection or referral biases than persons presenting to a single endoscopy unit. The consistency of the findings between the groups also decreases the possibility that referral bias for endoscopy alone explains the results. Second, the GERD-free control group was from a large regional population. Prior studies suggest the Kaiser Permanente membership closely approximates the region's underlying census demographics; (28) thus, the control group is more likely to approximate the prevalence of $H$. pylori in the general population than would a study using endoscopy controls (see the introduction section). Third, the pharmacy records permitted an evaluation for prior $\mathrm{H}$. pylori treatment for up to 7-10 years prior to the index date, decreasing the potential for bias from prior eradication. $(39,58)$ Finally, the data was of high quality and contained information for multiple potential confounders, including socioeconomic status. Measurements used trained personnel, a systematic protocol, an established laboratory, validated questionnaires, and comprehensive pharmacology databases.

There are several potential limitations of this analysis. First, observational studies cannot definitively establish cause and effect; however, since $H$. pylori colonization most commonly begins in childhood and GERD does not usually present until adulthood, at the minimum, the exposure (H. pylori) likely preceded the outcome (GERD) and randomized trials of H. pylori infection are not feasible.(47) Second, observational studies are subject to bias. We cannot exclude incomplete control of confounding or a component of reverse causation, whereby patients with GERD symptoms received prior treatment for $\mathrm{H}$. pylori; however, analyses for reverse causation using pharmacy records did not support this as a major bias. Third, although the members of the physician-assigned GERD group were randomly selected from among all GERD patients who underwent endoscopy during the study period, persons with GERD who undergo endoscopy may differ from the general population of GERD patients. Similarly, the GERD group excluded persons with Barrett's esophagus and was matched by age and gender to persons with Barrett's esophagus. The GERD population in this study may thus be somewhat older and have a higher proportion of males than a random sample of all GERD patients. However, the supplementary analysis of GERD vs. non-GERD patients confined to the control group (which would not be susceptible to referral biases for endoscopy) also demonstrated an inverse association between $\mathrm{H}$. pylori and GERD, and only a small minority of these subjects reported undergoing a prior endoscopy. The finding of a similar association in both groups provides support for the primary results. Third, nonresponders may lead to bias; however, electronic data suggested that nonresponders did not differ markedly from responders on several demographic variables.(29) This finding, combined with the absence of major confounding factors in the primary analyses, does not provide evidence for nonresponse bias.

In summary, we found strong inverse associations between the presence of $H$. pylori antibodies, GERD symptoms, and a diagnosis of GERD in a community-based population. These data are consistent with the hypothesis that the absence of Helicobacter pylori infection may be associated with GERD symptoms and GERD complications (such as esophagitis). Future studies are needed to evaluate whether eradication of $H$. pylori infection in childhood or early adulthood is associated with an increase in the risk of subsequent GERD, and longer term studies of eradication in adults.

\section{Acknowledgments}

Dr. Corley had full access to all of the data in the study and takes responsibility for the integrity of the data and the accuracy of the data analysis. 


\section{REFERENCES}

1. Beck IT, Champion MC, Lemire S, et al. The Second Canadian Consensus Conference on the Management of Patients with Gastroesophageal Reflux Disease. Canadian Journal of Gastroenterology. 1997; 11(Suppl B):7B-20B.

2. Kahrilas PJ. Gastroesophageal reflux disease. Jama. 1996; 276:983-8. [PubMed: 8805734]

3. Revicki DA, Wood M, Maton PN, et al. The impact of gastroesophageal reflux disease on healthrelated quality of life. Am J Med. 1998; 104:252-8. [PubMed: 9552088]

4. Lagergren J, Bergstrom R, Lindgren A, et al. Symptomatic gastroesophageal reflux as a risk factor for esophageal adenocarcinoma. N Engl J Med. 1999; 340:825-31. [PubMed: 10080844]

5. Koike T, Ohara S, Sekine H, et al. Helicobacter pylori infection inhibits reflux esophagitis by inducing atrophic gastritis. Am J Gastroenterol. 1999; 94:3468-72. [PubMed: 10606305]

6. Vicari JJ, Peek RM, Falk GW, et al. The seroprevalence of cagA-positive Helicobacter pylori strains in the spectrum of gastroesophageal reflux disease. Gastroenterology. 1998; 115:50-7. [PubMed: 9649458]

7. Feldman M, Cryer B, Sammer D, et al. Influence of H. pylori infection on meal-stimulated gastric acid secretion and gastroesophageal acid reflux. American Journal of Physiology. 1999; 277:G1159-64. [PubMed: 10600812]

8. Fallone CA, Barkun AN, Geottke MU, et al. Association of Helicobacter pylori genotype with gastroesophageal reflux disease and other upper gastrointestinal diseases. American Journal of Gastroenterology. 2000; 95:659-69. [PubMed: 10710054]

9. Moayyedi P, Bardhan C, Young L, et al. Helicobacter pylori eradication does not exacerbate reflux symptoms in gastroesophageal reflux disease. Gastroenterology. 2001; 121:1120-6. [PubMed: 11677204]

10. Laine L, Sugg J. Effect of Helicobacter pylori eradication on development of erosive esophagitis and gastroesophageal reflux disease symptoms: a post hoc analysis of eight double blind prospective studies. Am J Gastroenterol. 2002; 97:2992-7. [PubMed: 12492181]

11. Laine L, Dhir V. Helicobacter pylori eradication does not worsen quality of life related to reflux symptoms: a prospective trial. Aliment Pharmacol Ther. 2002; 16:1143-8. [PubMed: 12030957]

12. Ferrandez A, Benito R, Arenas J, et al. CagA-positive Helicobacter pylori infection is not associated with decreased risk of Barrett's esophagus in a population with high $\mathrm{H}$. pylori infection rate. BMC Gastroenterol. 2006; 6:7. [PubMed: 16483364]

13. Nordenstedt H, Nilsson M, Johnsen R, et al. Helicobacter pylori infection and gastroesophageal reflux in a population-based study (The HUNT Study). Helicobacter. 2007; 12:16-22. [PubMed: 17241296]

14. Cremonini F, Di Caro S, Delgado-Aros S, et al. Meta-analysis: the relationship between Helicobacter pylori infection and gastro-oesophageal reflux disease. Aliment Pharmacol Ther. 2003; 18:279-89. [PubMed: 12895212]

15. Raghunath A, Hungin AP, Wooff D, et al. Prevalence of Helicobacter pylori in patients with gastro-oesophageal reflux disease: systematic review. Bmj. 2003; 326:737. [PubMed: 12676842]

16. Johansson J, Hakansson HO, Mellblom L, et al. Risk factors for Barrett's oesophagus: a population-based approach. Scand J Gastroenterol. 2007; 42:148-56. [PubMed: 17327933]

17. Wong A, Fitzgerald RC. Epidemiologic risk factors for Barrett's esophagus and associated adenocarcinoma. Clin Gastroenterol Hepatol. 2005; 3:1-10. [PubMed: 15645398]

18. Zhang J, Chen XL, Wang KM, et al. Relationship of gastric Helicobacter pylori infection to Barrett's esophagus and gastro-esophageal reflux disease in Chinese. World J Gastroenterol. 2004; 10:672-5. [PubMed: 14991936]

19. Weston AP, Badr AS, Topalovski M, et al. Prospective evaluation of the prevalence of gastric Helicobacter pylori infection in patients with GERD, Barrett's esophagus, Barrett's dysplasia, and Barrett's adenocarcinoma. Am J Gastroenterol. 2000; 95:387-94. [PubMed: 10685740]

20. Vaezi MF, Falk GW, Peek RM, et al. CagA-positive strains of Helicobacter pylori may protect against Barrett's esophagus. Am J Gastroenterol. 2000; 95:2206-11. [PubMed: 11007219] 
21. Lord RV, Frommer DJ, Inder S, et al. Prevalence of Helicobacter pylori infection in 160 patients with Barrett's oesophagus or Barrett's adenocarcinoma. Australian and New Zealand Journal of Surgery. 2000 Report No.: 70.

22. Csendes A, Smok G, Cerda G, et al. Prevalence of Helicobacter pylori infection in 190 control subjects and in 236 patients with gastroesophageal reflux, erosive esophagitis or Barrett's esophagus. Dis Esophagus. 1997; 10:38-42. [PubMed: 9079272]

23. Nelson DB, Murdoch M, Sandozi IK, et al. Dyspepsia is associated with CagA-positive Helicobacter pylori. Am J Gastroenterol. 2000; 95:3412-7. [PubMed: 11151870]

24. Danesh J, Lawrence M, Murphy M, et al. Systematic review of the epidemiological evidence on Helicobacter pylori infection and nonulcer or uninvestigated dyspepsia. Arch Intern Med. 2000; 160:1192-8. [PubMed: 10789614]

25. Graham DY, Lew GM, Klein PD, et al. Effect of treatment of Helicobacter pylori infection on the long-term recurrence of gastric or duodenal ulcer. A randomized, controlled study. Ann Intern Med. 1992; 116:705-8. [PubMed: 1558340]

26. Huang JQ, Zheng GF, Sumanac K, et al. Meta-analysis of the relationship between cagA seropositivity and gastric cancer. Gastroenterology. 2003; 125:1636-44. [PubMed: 14724815]

27. Lee J, O'Morain C. Who should be treated for Helicobacter pylori infection? A review of consensus conferences and guidelines. Gastroenterology. 1997; 113:S99-106. [PubMed: 9394769]

28. Krieger N. Overcoming the absence of socioeconomic data in medical records: validation and application of a census-based methodology. American Journal of Public Health. 1992; 82:703-10. [PubMed: 1566949]

29. Corley DA, Kubo A, Levin TR, et al. Abdominal Obesity and Body Mass Index as Risk Factors for Barrett's Esophagus. Gastroenterology. 2007; 133:34-41. [PubMed: 17631128]

30. Rothman, KJ.; Greenland, S. Modern Epidemiology. Second ed.. Lippincott-Raven; Philadelphia: 1998.

31. Parsonnet J, Hansen S, Rodriguez L, et al. Helicobacter pylori infection and gastric lymphoma. N Engl J Med. 1994; 330:1267-71. [PubMed: 8145781]

32. Parsonnet J, Friedman GD, Vandersteen DP, et al. Helicobacter pylori infection and the risk of gastric carcinoma. N Engl J Med. 1991; 325:1127-31. [PubMed: 1891020]

33. Parsonnet J, Replogle M, Yang S, et al. Seroprevalence of CagA-positive strains among Helicobacter pylori-infected, healthy young adults. J Infect Dis. 1997; 175:1240-2. [PubMed: 9129095]

34. Parsonnet J, Friedman GD, Orentreich N, et al. Risk for gastric cancer in people with CagA positive or CagA negative Helicobacter pylori infection. Gut. 1997; 40:297-301. [PubMed: 9135515]

35. Locke GR, Talley NJ, Weaver AL, et al. A new questionnaire for gastroesophageal reflux disease. Mayo Clin Proc. 1994; 69:539-47. [PubMed: 8189759]

36. Breslow, NE.; Day, NE. Volume 1 - The analysis of case-control studies. International Agency for Research on Cancer; Lyon: 1980. Statistical methods in cancer research.

37. Kleinbaum, DG.; Kupper, LL.; Morgenstern, H. Principles and Quantitative Methods. Van Nostrand Reinhold; New York: 1982. Epidemiologic Research.

38. Hosmer, DW.; Lemeshow, S. Applied Logistic Regression. Second ed.. John Wiley \& Sons; New York: 2000.

39. Cutler AF, Prasad VM, Santogade P. Four-year trends in Helicobacter pylori IgG serology following successful eradication. Am J Med. 1998; 105:18-20. [PubMed: 9688016]

40. Diomedi M, Pietroiusti A, Silvestrini M, et al. CagA-positive Helicobacter pylori strains may influence the natural history of atherosclerotic stroke. Neurology. 2004; 63:800-4. [PubMed: 15365126]

41. Ye W, Held M, Lagergren J, et al. Helicobacter pylori infection and gastric atrophy: risk of adenocarcinoma and squamous-cell carcinoma of the esophagus and adenocarcinoma of the gastric cardia. J Natl Cancer Inst. 2004; 96:388-96. [PubMed: 14996860]

42. Wu JC, Sung JJ, Chan FK, et al. Helicobacter pylori infection is associated with milder gastrooesophageal reflux disease. Aliment Pharmacol Ther. 2000; 14:427-32. [PubMed: 10759622] 
43. Labenz J, Jaspersen D, Kulig M, et al. Risk factors for erosive esophagitis: a multivariate analysis based on the ProGERD study initiative. Am J Gastroenterol. 2004; 99:1652-6. [PubMed: 15330897]

44. Koike T, Ohara S, Sekine H, et al. Helicobacter pylori infection prevents erosive reflux oesophagitis by decreasing gastric acid secretion. Gut. 2001; 49:330-4. [PubMed: 11511552]

45. Corley DA, Kubo A, Levin TR, et al. Helicobacter Pylori Infection and the Risk of Barrett's Oesophagus: A Community-Based Study. Gut. 2007

46. Malaty HM, El-Kasabany A, Graham DY, et al. Age at acquisition of Helicobacter pylori infection: a follow-up study from infancy to adulthood. Lancet. 2002; 359:931-5. [PubMed: 11918912]

47. Rowland M, Daly L, Vaughan M, et al. Age-specific incidence of Helicobacter pylori. Gastroenterology. 2006; 130:65-72. quiz 211. [PubMed: 16401469]

48. Webb PM, Crabtree JE, Forman D, The Eurogst Study Group. Gastric cancer, cytotoxin-associated gene A-positive Helicobacter pylori, and serum pepsinogens: an international study. Gastroenterology. 1999; 116:269-76. [PubMed: 9922306]

49. Oksanen A, Sipponen P, Karttunen R, et al. Atrophic gastritis and Helicobacter pylori infection in outpatients referred for gastroscopy. Gut. 2000; 46:460-3. [PubMed: 10716672]

50. Kamangar F, Dawsey SM, Blaser MJ, et al. Opposing risks of gastric cardia and noncardia gastric adenocarcinomas associated with Helicobacter pylori seropositivity. J Natl Cancer Inst. 2006; 98:1445-52. [PubMed: 17047193]

51. Wu AH, Crabtree JE, Bernstein L, et al. Role of Helicobacter pylori CagA+ strains and risk of adenocarcinoma of the stomach and esophagus. Int J Cancer. 2003; 103:815-21. [PubMed: 12516104]

52. de Martel C, Llosa AE, Farr SM, et al. Helicobacter pylori Infection and the Risk of Development of Esophageal Adenocarcinoma. J Infect Dis. 2005; 191:761-7. [PubMed: 15688293]

53. Chow WH, Blaser MJ, Blot WJ, et al. An inverse relation between cagA+ strains of Helicobacter pylori infection and risk of esophageal and gastric cardia adenocarcinoma. Cancer Res. 1998; 58:588-90. [PubMed: 9485003]

54. Everhart JE, Kruszon-Moran D, Perez-Perez G. Reliability of Helicobacter pylori and CagA serological assays. Clin Diagn Lab Immunol. 2002; 9:412-6. [PubMed: 11874887]

55. Suzuki G, Cullings H, Fujiwara S, et al. Low-positive antibody titer against Helicobacter pylori cytotoxin-associated gene A (CagA) may predict future gastric cancer better than simple seropositivity against H. pylori CagA or against H. pylori. Cancer Epidemiol Biomarkers Prev. 2007; 16:1224-8. [PubMed: 17548689]

56. Delaney B, McColl K. Review article: Helicobacter pylori and gastro-oesophageal reflux disease. Aliment Pharmacol Ther. 2005; 22(Suppl 1):32-40. [PubMed: 16042657]

57. McColl KE, el-Omar E, Gillen D. Interactions between H. pylori infection, gastric acid secretion and anti-secretory therapy. Br Med Bull. 1998; 54:121-38. [PubMed: 9604437]

58. Gisbert JP, Blanco M, Benito LM, et al. Value of quantitative serology for confirmation of Helicobacter pylori eradication: an 18-month follow-up study. Clin Infect Dis. 2000; 30:976-80. [PubMed: 10880321] 


\section{Table1}

Characteristics of study groups

\begin{tabular}{|c|c|c|c|}
\hline & GERD Group (physician-assigned) & $\begin{array}{c}\text { Population Group with } \\
\text { GERD }\end{array}$ & $\begin{array}{c}\text { Population controls without } \\
\text { GERD }\end{array}$ \\
\hline & Number (\%) & Number (\%) & Number or Mean (\%) \\
\hline Number of subjects & 301 & 81 & 175 \\
\hline Age (years) & & & $9(5)$ \\
\hline $20-39$ & $12(4)$ & $0(0)$ & $60(34)$ \\
\hline $40-59$ & $110(37)$ & $25(31)$ & $106(61)$ \\
\hline $60-79$ & $179(59)$ & $56(69)$ & $148(85)$ \\
\hline Race/Ethnicity & & & $9(5)$ \\
\hline White & $242(80)$ & $68(84)$ & $7(4)$ \\
\hline Black & $17(6)$ & $4(5)$ & $6(3)$ \\
\hline Hispanic & $20(7)$ & $3(4)$ & $5(3)$ \\
\hline Asian & $11(4)$ & $5(6)$ & $0(0)$ \\
\hline Others & $9(3)$ & $0(0)$ & $116(66)$ \\
\hline Unknown & $2(1)$ & $1(1)$ & $79(45)$ \\
\hline Male & $207(69)$ & $58(72)$ & $54(67)$ \\
\hline Smoking status (Ever smoked) & $175(58)$ & & \\
\hline
\end{tabular}

The groups were frequency matched by sex, age (by 5 year age groups) and center of diagnosis (see methods section). 
Table 2

The associations between antibody status for Helicobacter pylori infection and a physician-defined GERD diagnosis, stratified by cagA antibody status

\begin{tabular}{|l|l|c|c|}
\hline & & Number of Subjects & $\begin{array}{c}\text { Odds Ratio } \mathbf{1} \text { (95\% Confidence } \\
\text { Interval) }\end{array}$ \\
\hline $\begin{array}{l}\text { Helicobacter pylori Antibody } \\
\text { Status }\end{array}$ & CagA+ Antibody Status & $\begin{array}{c}\text { GERD patients (physician defined)/ } \\
\text { Controls }\end{array}$ & GERD vs. Controls \\
\hline \multirow{2}{*}{ Negative } & Negative & $259 / 130$ & 1.00 \\
\cline { 2 - 4 } & Positive & $13 / 1$ & $5.63(0.72-44.29)$ \\
\hline \multirow{2}{*}{ Positive } & Negative & $19 / 27$ & $0.25(0.13-0.49)$ \\
\cline { 2 - 4 } & Positive & $10 / 17$ & $0.26(0.11-0.62)$ \\
\hline Any CagA+ subjects ${ }^{2}$ & & $7 / 18$ & $0.58(0.29-1.14)$ \\
\hline All H.Pylori Positive subjects & & $29 / 44$ & $0.27(0.15-0.47)$ \\
\hline
\end{tabular}

${ }^{l}$ Odds ratios were adjusted for gender, age, facility of diagnosis, and educational status.

2 These include both H. pylori positive and H. pylori negative subjects.

3 These include both cagA positive and cagA negative subjects. 
Table 3

Helicobacter pylori status and GERD Symptom Severity (Physician-defined GERD group vs. population controls with heartburn or acid regurgitation less than once a month or never)

\begin{tabular}{|c|c|c|c|c|}
\hline & & Number of Subjects 1 & 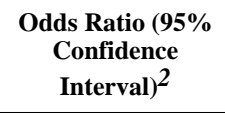 & $\begin{array}{c}\text { Odds Ratio }(95 \% \\
\text { Confidence Interval) }\end{array}$ \\
\hline & & & $\begin{array}{c}\text { H. pylori antibody } \\
\text { positive }^{3}\end{array}$ & CagA antibody positive \\
\hline Population controls & No GERD ${ }^{5}$ & 175 & 1.0 & 1.0 \\
\hline \multirow[t]{9}{*}{ Physician-defined GERD patients } & GERD Characteristic & 301 & $0.27(0.15-0.47)$ & $0.26(0.11-0.61)$ \\
\hline & Severity & & & \\
\hline & Mild & 33 & $0.45(0.16-1.26)$ & $0.19(0.02-1.59)$ \\
\hline & Moderate & 118 & $0.19(0.08-0.44)$ & $0.25(0.08-0.79)$ \\
\hline & Severe or very severe & 134 & $0.18(0.08-0.41)$ & $0.20(0.06-0.68)$ \\
\hline & Frequency & & & \\
\hline & Monthly & 33 & $0.31(0.09-1.01)$ & $0.21(0.03-1.75)$ \\
\hline & Weekly & 59 & $0.15(0.05-0.48)$ & $0.10(0.01-0.82)$ \\
\hline & $\begin{array}{l}\text { Several times a week or } \\
\text { daily }\end{array}$ & 166 & $0.18(0.09-0.38)$ & $0.24(0.09-0.68)$ \\
\hline
\end{tabular}

The sum of the subcategories may not equal the total for the entire category due to missing values or other values for a subcategory. The totals exclude population controls with greater than monthly (but less than weekly) GERD symptoms.

2 Odds ratios were adjusted for gender, age, facility of diagnosis, and educational status.

3 "Exposed" subjects were positive for $H$. pylori antibody; this included both cagA positive and cagA negative subjects. "Unexposed" subjects were H. pylori negative and cagA negative.

4 "Exposed" subjects were positive for both $H$. pylori antibody positive and cagA antibody. "Unexposed" subjects were $H$. pylori negative and cagA negative.

5

Heartburn or acid regurgitation less than once a month or never. 


\section{Table 4}

The associations between antibody status for Helicobacter pylori infection and a self-described GERD symptoms (among the population controls), stratified by cagA antibody status

\begin{tabular}{|l|l|c|c|}
\hline & & Number of Subjects & $\begin{array}{c}\text { Odds Ratio } \mathbf{1} \text { (95\% Confidence } \\
\text { Interval) }\end{array}$ \\
\hline $\begin{array}{l}\text { Helicobacter pylori Antibody } \\
\text { Status }\end{array}$ & CagA+ Antibody Status & $\begin{array}{c}\text { GERD patients (symptom defined)/ } \\
\text { Controls }\end{array}$ & GERD vs. Controls \\
\hline \multirow{2}{*}{ Negative } & Negative & $64 / 130$ & 1.00 \\
\cline { 2 - 4 } & Positive & $2 / 1$ & $7.02(0.53-92.90)$ \\
\hline \multirow{2}{*}{ Positive } & Negative & $10 / 27$ & $0.42(0.18-1.00)$ \\
\cline { 2 - 4 } & Positive & $5 / 17$ & $0.41(0.14-1.23)$ \\
\hline Any CagA+ subjects ${ }^{2}$ & & $7 / 18$ & $0.62(0.24-1.62)$ \\
\hline All H.Pylori Positive subjects & & $15 / 44$ & $0.46(0.23-0.95)$ \\
\hline
\end{tabular}

${ }^{l}$ Odds ratios were adjusted for gender, age, facility of diagnosis, and educational status.

2 These include both $H$. pylori positive and H. pylori negative subjects.

3 These include both cagA positive and cagA negative subjects. 